\title{
Tuberculosis Infection in a Patient with Lung Cancer under PD-L1 Inhibition: A Case Report
}

\author{
Eleni Papadaki1 ${ }^{*}$, Manika Katerina1, Boutis Anastasios' ${ }^{2}$, Kioumis Ioannis ${ }^{3}$, \\ Kontakiotis Theodoros ${ }^{1}$
}

\author{
${ }^{1}$ Respiratory Medicine, Medical School, Aristotle University of Thessaloniki, Pulmonary Department, “G. Papanikolaou” Hospital, \\ Thessaloniki, Greece \\ ${ }^{2}$ Oncology, 1st Department of Medical Oncology, Theagenio Cancer Hospital, Thessaloniki, Greece \\ ${ }^{3}$ Respiratory Medicine and Infectious Diseases, Medical School, Aristotle University of Thessaloniki, Respiratory Failure \\ Department, “G. Papanikolaou” Hospital, Thessaloniki, Greece \\ Email: ^elenipd@hotmail.com, ktmn05@yahoo.gr, alboutis@otenet.gr, ikioum@yahoo.gr,kontak@auth.gr
}

How to cite this paper: Papadaki, E., Katerina, M., Anastasios, B., Ioannis, K. and Theodoros, K. (2020) Tuberculosis Infection in a Patient with Lung Cancer under PD-L1 Inhibition: A Case Report. Journal of Tuberculosis Research, 8, 158-164. https://doi.org/10.4236/jtr.2020.83014

Received: July 9, 2020

Accepted: August 17, 2020

Published: August 20, 2020

Copyright $\odot 2020$ by author(s) and Scientific Research Publishing Inc. This work is licensed under the Creative Commons Attribution International License (CC BY 4.0).

http://creativecommons.org/licenses/by/4.0/

\begin{abstract}
Background: Immune checkpoint inhibitors have made a great novelty in the treatment of various cancer types, showing favourable outcomes and good tolerance by cancer patients. Immune checkpoint inhibitors enhance and promote anti-tumor immunity, which can result in a wide range of adverse events, termed as immune-related adverse events, which are characterized by excessive immunity response. Although immune related adverse events are not considered to be originated from infectious causes, cases of cancer patients developing active tuberculosis during treatment with immune checkpoint inhibitors have been reported. Aim: The aim of the current case report is to highlight the importance of including opportunistic infections, such as tuberculosis, in the differential diagnosis of complications in the treatment of cancer patients receiving immunotherapy. Case presentation: This case report describes a 62-year-old Caucasian male patient who developed active pulmonary tuberculosis after treatment with Durvalumab, an anti-programmed death cell ligand-1 antibody, administered as therapy for non small cell lung cancer. The diagnosis was challenging because of the fact that the clinical presentation and the radiographic imaging were compatible with disease progression. Conclusion: Screening for active or latent tuberculosis should be part of everyday practice before the initial administration of immunotherapy in oncologic patients.
\end{abstract}

\section{Keywords}

Tuberculosis, Immune Checkpoint Inhibitors, Durvalumab, Immunotherapy, PD-L1 


\section{Introduction}

In the last decade, a major development has been noted in oncology with the use of immune checkpoint inhibitors (ICIs) in the treatment of various cancer types. Although ICIs are in general well tolerated by patients compared to cytotoxic therapy, immune-related adverse events (irAEs) have been reported, involving different systems of the body [1]. Most of irAEs are a result of immunoactivation and are treated with immunosuppression with the use of corticosteroids and most of them are not related with infectious diseases. However, cases of development of active tuberculosis (TB) during treatment with ICIs are becoming increasingly frequent. According to World Health Organization (WHO), TB remains a major cause of morbidity and mortality worldwide [2]. Although the prevalence of TB is much higher among immunosuppressed patients compared to immunosufficient ones, screening for TB among cancer patients has not yet been established. Given the fact that in cancer patients the clinical presentation and the radiographic images can be atypical and misleading, it is crucial that TB is involved in the differential diagnosis of the complications of cancer therapy [3]. We report a patient with non small cell lung cancer (NSCLC), who developed active pulmonary tuberculosis, few months after treatment with Durvalumab, an anti-programmed death cell ligand-1 (anti-PD-L1) antibody, which is used as monotherapy in locally advanced stage III NSCLC after receiving chemoradiotherapy [4]. To our knowledge, there is currently no report of active TB developing as an irAE with the specific regimen.

\section{Case Presentation}

A 62-year-old male patient was diagnosed with clinical stage IIIA lung adenocarcinoma in June 2017. Chest computed tomography (CT) at the time of diagnosis showed a $4 \mathrm{~cm}$ mass in the right upper lobe, right hilar and right upper paratracheal lymphadenopathy (Figure 1(a) and Figure 1(b)). The patient received concurrent chemotherapy and radiotherapy for three months, followed by immunotherapy with Durvalumab from January until August 2018. At that point, a significant radiographic improvement was noted and one month later the patient underwent right upper lobectomy followed by 3 cycles of adjuvant chemotherapy. The postoperative chest CT of the patient showed no residual disease, with only post radiation lesions remaining in the superior segment of the right lower lobe (Figure 1(c) and Figure 1(d)). Five months later, the patient developed fever, fatigue and weight loss. Extensive infiltrates in the middle lobe and the superior segment of the right lower lobe were seen on his chest computed tomography. A PET CT was performed, revealing hypermetabolic tissue corresponding to the infiltrates of the CT (SUVmax 19.4) (Figures 2(a)-(d)). The findings of the PET CT were consistent with progressive disease and the patient underwent bronchoscopy in July 2019. No abnormal findings were detected during bronchoscopy and blind biopsies from the middle lobe were collected. The biopsies and the cytologic examination of the bronchoalveolar fluid (BAL) were negative for malignancy and the patient was referred for 


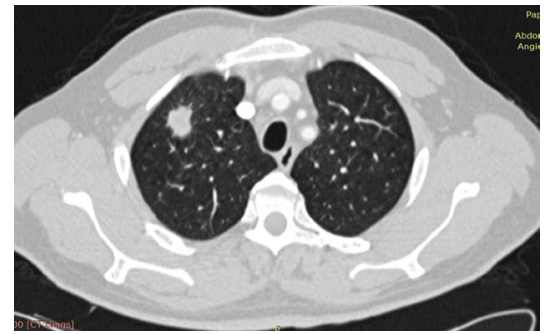

(a)

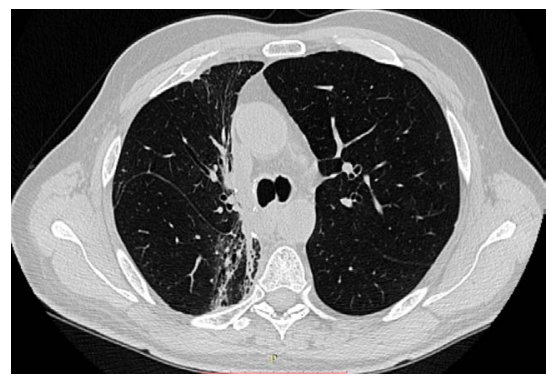

(c)

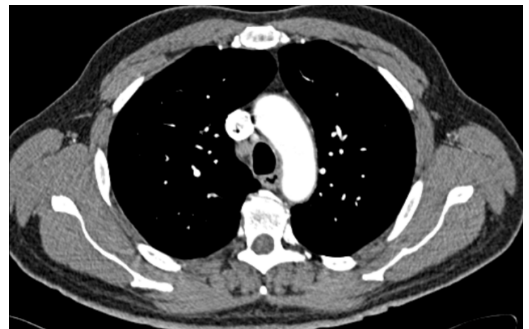

(b)

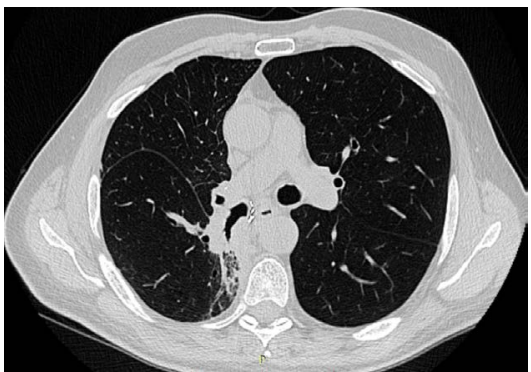

(d)

Figure 1. (a)-(d) Chest CT at the time of initial diagnosis revealing a $4 \mathrm{~cm}$ mass in the right upper lobe, right hilar and right upper paratracheal lymphadenopathy ((a) and (b)). Postoperative chest CT showing no residual disease, with only post radiation lesions remaining in the superior segment of the right lower lobe ((c) and (d)).

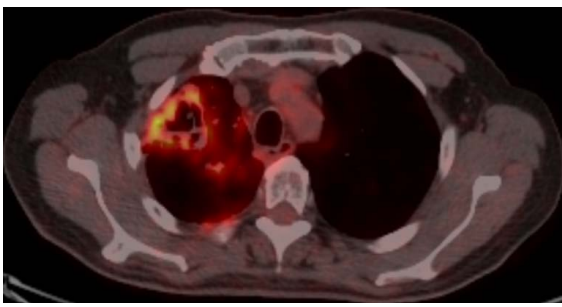

(a)

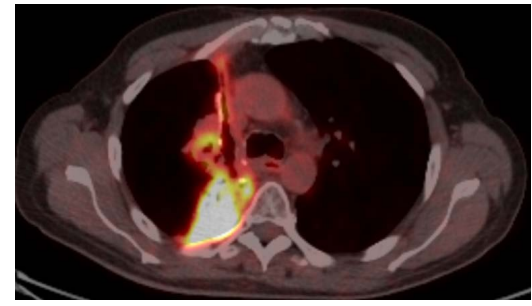

(c)

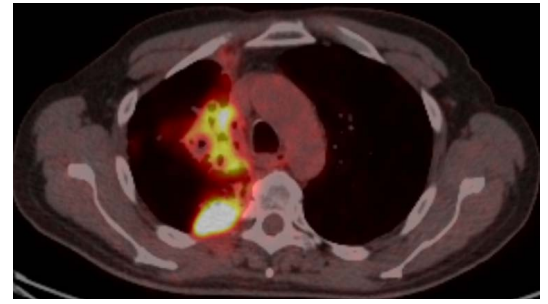

(b)

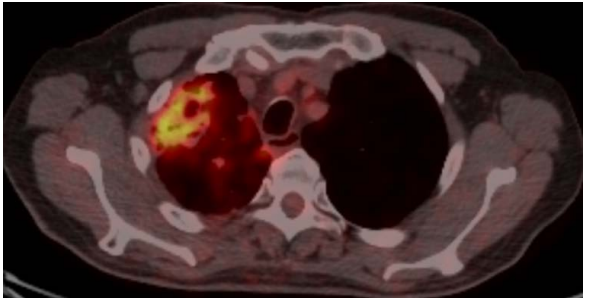

(d)

Figure 2. (a)-(d) PET CT showing extensive hypermetabolic infiltrates (SUV 19,4) in the middle lobe and the superior segment of the right lower lobe, consistent with disease progression.

further investigation by CT guided fine needle biopsy (FNB). However, a new CT scan showed further deterioration with cavity formation and infiltrates and nodules seen in both lungs (Figures 3(a)-(d)). The patient kept being febrile and continued to complain about fatigue. He was admitted to hospital twice, where he was given two courses of advanced antibiotics with little clinical improvement, but with remission of the symptoms some days after the cessation of the 
antibiotics. Three weeks after bronchoscopy, M. tuberculosis was identified in BAL culture, sensitive to all antituberculous drugs. Consequently a four-drug antituberculous regimen was initiated. The patient responded favourably with a great clinical and radiographic improvement (Figures 4(a)-(d)) and showed good tolerance to treatment. While he was on the fourth month of anti-TB therapy, the patient developed brain metastases consistent with progressive disease and died in March 2020. Informed consent was obtained from the patient's wife for the publication of the case report.

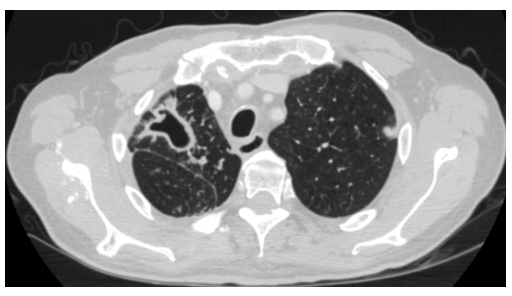

(a)

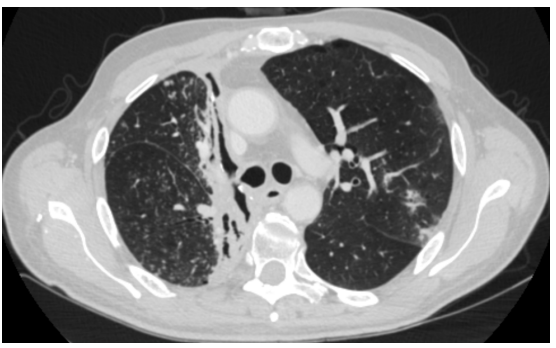

(c)

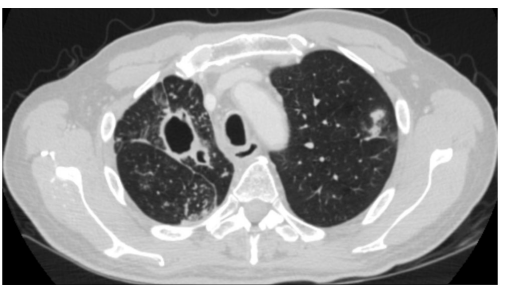

(b)

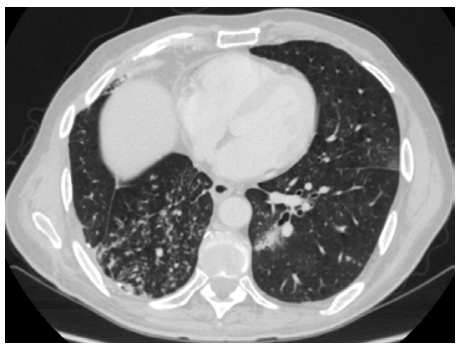

(d)

Figure 3. (a)-(d) Chest CT in July 2019 showing cavity formation, infiltrates and nodules seen in both lungs $((\mathrm{a})-(\mathrm{d}))$.

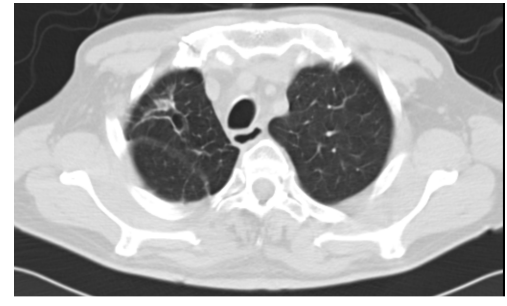

(a)

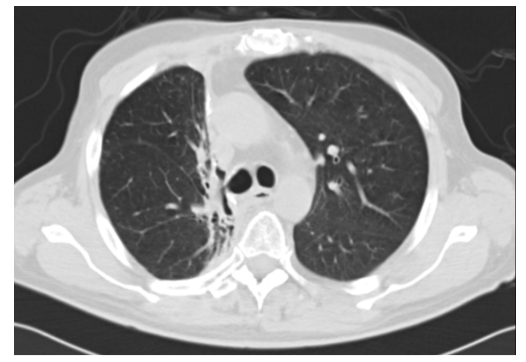

(c)

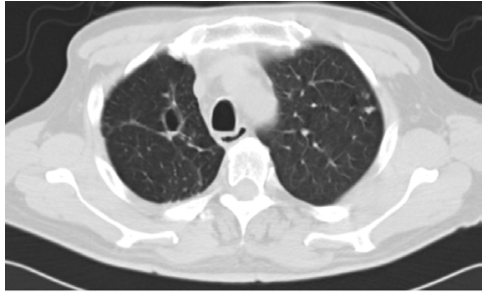

(b)

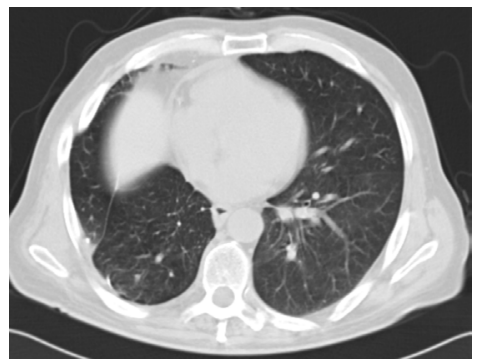

(d)

Figure 4. (a)-(d) Chest CT 3 months after the initiation of anti-TB therapy showing a significant radiographic improvement with a great reduction of the size and thickness of the cavity, as well as the number of the nodules ((a)-(d)). 


\section{Discussion}

Durvalumab is an immune checkpoint inhibitor which was first approved for urothelial carcinoma and NSCLC in 2017. It is an IgG1 human antibody, targeting programmed-cell death ligand-1 (PD-L1) and consequently inhibiting the PD-1/PD-L1 pathway [5]. Normally, activation of PD1/PD-L1 pathway leads to suppression of T-cell activation. Tumor cells in many cancer types express PD-1 and in that way escape immune surveillance. Blockage of PD-1/PDL-1 pathway leads to T-cell activation against tumor cells and consequently immune functions are enhanced for the benefit of the host. Antibody immune checkpoint inhibitors have so far demonstrated a satisfying tolerance and the side effects are remarkably fewer compared to cytotoxic agents. The most common irAEs include endocrine (hypo/hyperthyroidism), gastrointestinal (colitis, diarrhoea), lung (pneumonitis), skin and musculoskeletal disorders [1]. ICIs enhance immune functions and therefore irAEs are not expected to include infectious diseases. Moreover, a study that was conducted by J. Jurado et al. showed that impaired immunity in TB is associated with suppressed T-cell activation and reduced production of IFN- $\gamma$ mediated by PD-1/PD-L1 pathway, suggesting that blockage of PD-1/PD-L1 pathway plays a protective role in M. tuberculosis infection [6]. Despite this fact, PD-1 deficient mice were found to be extremely susceptible to tuberculosis infection, showing reduced survival and higher bacterial loads, due to altered inflamatory response [7]. As described by Lázár-Molnár et al., the PD-1/PD-L1 pathway is essential in order to control excessive inflammatory response against $M$. tuberculosis, implying that excessive immunity could be as harmful as impaired immunity. Indeed, our patient showed a dramatic deterioration of his CT scan due to TB after surgery, consistent with increased inflammation, which may be relevant to the previous administration of Durvalumab.

In clinical practice, it is already known that the risk of developing active tuberculosis during cytotoxic chemotherapy is high, due to immune suppression. Recently, there are data suggesting that active pulmonary or extrapulmonary tuberculosis can also be detected with the use of immunotherapy in cancer. According to a multicentre, prospective registry in France, the relative incidence of tuberculosis among cancer patients receiving anti-PD-1/PD-L1 agents could be about one case per 1000 patients, which is much higher than the incidence of TB in the general population [8]. In review of current literature there are five cases of tuberculosis (three pulmonary, one pericardial, one spinal) among patients receiving Nivolumab for NSCLC [8] [9] [10] [11] [12]. Four cases of patients under therapy with Pembrolizumab for melanoma [8] [13] [14] and Hodgkin's lymphoma [15] have also been published. Three patients developed pulmonary tuberculosis and the fourth pleural tuberculosis. Our patient was diagnosed with pulmonary tuberculosis a few months after therapy with Durvalumab, which, to our knowledge, is the first case of TB reported for the specific drug. Only one other case diagnosed with invasive aspergillosis while being under treatment 
with Durvalumab for NSCLC has been reported [16]. Our patient, like most cases mentioned above, had also received chemotherapy in addition to Durvalumab and therefore it is difficult to associate the activation of TB with the administration of Durvalumab alone. However, the dramatic clinical and radiographic presentation as well as the rapid progress of the disease could be connected with the excessive inflammation.

\section{Conclusion}

In conclusion, clinical doctors should be highly suspicious when dealing with oncologic patients receiving anti-PD-1/PD-L1 inhibitors in respect of the wide range of the adverse events and the lack of experience so far. The impression that anti-PD-1/PD-L1 inhibitors are not associated with high prevalence of infectious diseases is challenged. Opportunistic infections, and especially tuberculosis, should be part of the differential diagnosis, particularly in cases with compatible clinical presentation. In patients diagnosed with $\mathrm{TB}$ while on treatment with anti-PD-1/PD-L1 inhibitors a rapid deterioration due to excessive inflammation may be anticipated. Screening for active or latent TB before the administration of ICIs to cancer patients should be considered prospectively.

\section{Conflicts of Interest}

The authors declare no conflicts of interest regarding the publication of this paper.

\section{References}

[1] Khoja, L., Day, D., et al. (2017) Tumor- and Class-Specific Patterns of Immune-Related Adverse Events of Immune Checkpoint Inhibitors: A Systematic Review. Annals of Oncology, 28, 2377-2385. https://doi.org/10.1093/annonc/mdx286

[2] World Health Organization (2019) Global Tuberculosis Report 2019. World Health Organization, Geneva!?

[3] WHO (2015) Systematic Screening for Active Tuberculosis. 146.

[4] Corrales, L., Scilla, K., Caglevic, C., Miller, K., Oliveira, J. and Rolfo, C. (2018) Immunotherapy in Lung Cancer: A New Age in Cancer Treatment. Advances in Experimental Medicine and Biology, 995, 65-95. https://doi.org/10.1007/978-3-030-02505-2 3

[5] Picardo, S.L., Doi, J. and Hansen, A.R. (2020) Structure and Optimization of Checkpoint Inhibitors. Cancers (Basel), 12, 1-15. https://doi.org/10.3390/cancers 12010038

[6] Jurado, J.O., Alvarez, I.B., Pasquinelli, V., Martínez, G.J., Quiroga, M.F., Abbate, E., et al. (2008) Programmed Death (PD)-1:PD-Ligand 1/PD-Ligand 2 Pathway Inhibits $\mathrm{T}$ Cell Effector Functions during Human Tuberculosis. The Journal of Immunology, 181, 116-125. https://doi.org/10.4049/jimmunol.181.1.116

[7] Lázár-Molnár, E., Chen, B., Sweeney, K.A., Wang, E.J., Liu, W., Lin, J., et al. (2010) Programmed Death-1 (PD-1)-Deficient Mice Are Extraordinarily Sensitive to Tuberculosis. Proceedings of the National Academy of Sciences of the United States of America, 107, 13402-13407. https://doi.org/10.1073/pnas.1007394107 
[8] Picchi, H., Mateus, C., Chouaid, C., Besse, B., Marabelle, A., Michot, J.M., et al. (2018) Infectious Complications Associated with the Use of Immune Checkpoint Inhibitors in Oncology: Reactivation of Tuberculosis after Anti PD-1 Treatment. Clinical Microbiology and Infection, 24, 216-218. https://doi.org/10.1016/j.cmi.2017.12.003

[9] Fujita, K., Terashima, T. and Mio, T. (2016) Anti-PD1 Antibody Treatment and the Development of Acute Pulmonary Tuberculosis. Journal of Thoracic Oncology, 11, 2238-2240. https://doi.org/10.1016/j.jtho.2016.07.006

[10] Chu, Y.C., Fang, K.C., Chen, H.C., Yeh, Y.C., Tseng, C.E., Chou, T.Y., et al. (2017) Pericardial Tamponade Caused by a Hypersensitivity Response to Tuberculosis Reactivation after Anti-PD-1 Treatment in a Patient with Advanced Pulmonary Adenocarcinoma. Journal of Thoracic Oncology, 12, e111-e114. https://doi.org/10.1016/j.jtho.2017.03.012

[11] Jensen, K.H., Persson, G., Bondgaard, A.L. and Pøhl, M. (2018) Development of Pulmonary Tuberculosis Following Treatment with Anti-PD-1 for Non-Small Cell Lung Cancer. Acta Oncologica (Madr), 57, 1127-1128. https://doi.org/10.1080/0284186X.2018.1433877

[12] van Eeden, R., Rapoport, B.L., Smit, T. and Anderson, R. (2019) Tuberculosis Infection in a Patient Treated with Nivolumab for Non-Small Cell Lung Cancer: Case Report and Literature Review. Frontiers in Oncology, 9, 659. https://doi.org/10.3389/fonc.2019.00659

[13] Elkington, P.T., Bateman, A.C., Thomas, G.J. and Ottensmeier, C.H. (2018) Implications of Tuberculosis Reactivation after Immune Checkpoint Inhibition. American Journal of Respiratory and Critical Care Medicine, 198, 1451-1453. https://doi.org/10.1164/rccm.201807-1250LE

[14] He, W., Zhang, X., Li, W., Kong, C., Wang, Y., Zhu, L., et al. (2018) Activated Pulmonary Tuberculosis in a Patient with Melanoma during PD-1 Inhibition: A Case Report. OncoTargets and Therapy, 11, 7423-7427.

https://doi.org/10.2147/OTT.S178246

[15] Lee, J.J.X., Chan, A. and Tang, T. (2016) Tuberculosis Reactivation in a Patient Receiving Anti-Programmed Death-1 (PD-1) Inhibitor for Relapsed Hodgkin's Lymphoma. Acta Oncologica (Madr), 55, 519-520. https://doi.org/10.3109/0284186X.2015.1125017

[16] Gupta, A., Tun, A., Ticona, K., Baqui, A. and Guevara, E. (2019) Invasive Aspergillosis in a Patient with Stage III (or 3a or 3b) Non-Small-Cell Lung Cancer Treated with Durvalumab. Case Reports in Oncological Medicine, 2019, Article ID: 2178925. https://doi.org/10.1155/2019/2178925 